\title{
Biomimetics in Action: Practical Applications of Single Layer Centrifugation for equine breeding
}

\section{Morrell JM}

Clinical Sciences, Swedish University of Agricultural Sciences, Box 7054, SE-75007 Uppsala, Sweden

\begin{abstract}
Single Layer Centrifugation (SLC) of spermatozoa through a species-specific colloid has been shown to be effective in selecting the best spermatozoa from stallion semen. The method is easier to use and less time-consuming than density gradient centrifugation (DGC), and has been scaled-up to allow whole ejaculates to be processed in a practical manner. The major applications for SLC-sperm selection are as follows: to improve sperm quality in artificial insemination (AI) doses, particularly for "problem" stallions; to increase the "shelf-life" of normal sperm samples, either by processing the fresh semen before preparing Al doses, or by processing the stored semen dose to extract the best spermatozoa; to circumvent the problem of spermatozoa that do not tolerate cooling to $4-6^{\circ} \mathrm{C}$; to improve cryosurvival by removing dead and dying spermatozoa prior to cryopreservation, or selecting the live spermatozoa post-thawing; to select morphologically normal spermatozoa with intact chromatin from sub-fertile stallion semen for ICSI, thus increasing the number of blastocysts; to remove pathogens (viruses, bacteria); to accelerate the process of flow cytometric sex selection by removing the dead and dying sperm before passage through the laser beam; to conserve rare breeds. These applications are discussed and practical examples provided.
\end{abstract}

Keywords: SLC; Androcoll-E; equine breeding; stallion semen

\section{Introduction}

Biomimetics, the use of technologies and/or processes that mimic a naturally occurring event, can be used to advantage in animal breeding [1]. Evidence for selection mechanisms within the female reproductive tract that permit only morphologically normal spermatozoa with intact membranes and good chromatin integrity to pass up to the site of fertilization ("good" spermatozoa) has been discussed frequently over the last two decades [2]. Some semen preparation techniques mimic this selection in vitro and thus fit the description of biomimetics. The purpose of this review is to introduce Single Layer Centrifugation (SLC) as one such biomimetic technique and to describe its potential applications in the equine artificial insemination (AI) industry.

SLC as a method of processing semen first made its debut when [3] reported their attempts to process human ejaculates using one layer of Percoll, rather than two or more layers of colloid of different densities, as in the conventional density gradient centrifugation (DGC). These techniques of DGC and SLC have been described in detail previously [1]. The results of Sharma et al. [3] showed that although it was possible to improve sperm quality in human ejaculates of low initial quality, no improvement in sperm quality was seen when "normal" human ejaculates were processed in this manner. Sieme et al. [4] obtained similar results using one layer of Percoll ${ }^{\mathrm{TM}}$ for stallion semen.

The first indication that sperm quality in "normal" stallion ejaculates could be improved by SLC was given in a preliminary study using Androcoll-E, a new species-specific colloid formulation for stallion spermatozoa [5]. This formulation incorporated silane-coated silica in an optimised salt solution instead of Percoll ${ }^{\mathrm{TM}}$ (polyvinylpyrrolidonecoated silica) to which various salt solutions had been added. The original SLC methodology was later modified to enable larger volumes of ejaculate to be processed without compromising sperm quality in the resulting preparations [6], a feature that was not possible with DGC without a drop in sperm yield [7]. The initial stage of preparing a scaledup SLC with Androcoll-E and stallion semen is shown in (Figure 1). It was possible to prepare an average gel-free stallion ejaculate using six to eight 50-mL centrifuge tubes using the SLC-Large method [6].
Although there have been numerous reports on the use of SLC to improve sperm quality, as shown by laboratory assays [8-13] there are few reports on fertility in the field. The purpose of this review, therefore, is to examine potential practical applications of SLC in the equine breeding industry and report preliminary fertility data for some of these applications.

\section{Advantages of SLC for preparing stallion sperm doses for AI}

The following benefits of using SLC to prepare animal spermatozoa have been reported previously:

i) SLC-selected stallion spermatozoa show better progressive motility, normal morphology, viability and chromatin integrity than their unselected counterparts $[8,9,14]$;

ii) Sperm quality (motility, viability and chromatin integrity) declines more slowly during cold storage than in unselected semen $[10,11,15]$;

iii) SLC-selected spermatozoa retain progressive motility when stored at room temperature (approximately $22^{\circ} \mathrm{C}$ ) for $48-72 \mathrm{~h}$ [8];

iv) SLC can be scaled-up to prepare whole stallion ejaculates without loss of sperm quality [6];

v) SLC-selected samples have better sperm quality than samples produced by "sperm washing" and retain this improved quality for longer [16];

Corresponding author: Morrell JM, Clinical Sciences, Swedish University of Agricultural Sciences, Box 7054, SE-75007 Uppsala, Sweden, E-mail: jane. morrell@kv.slu.se

Received September 02, 2011; Accepted September 20, 2011; Published September 26, 2011

Citation: Morrell JM (2011) Biomimetics in Action: Practical Applications of Single Layer Centrifugation for equine breeding. J Veterinar Sci Technol 2:107. doi:10.4172/2157-7579.1000107

Copyright: ( $) 2011$ Morrell JM. This is an open-access article distributed under the terms of the Creative Commons Attribution License, which permits unrestricted use, distribution, and reproduction in any medium, provided the original author and source are credited. 
vi) SLC does not adversely affect sperm capacitation status [13];

vii) SLC can be used post-thawing to select the motile, viable spermatozoa from cryopreserved semen doses [12,17];

viii) Spermatozoa can be separated from viruses in the semen (Blomqvist et al., unpublished data),

ix) Bacterial contamination can be removed from the ejaculate, thus potentially allowing a reduction in the use of antibiotics in semen extenders [17].

Since pregnancy rates in artificially inseminated mares have been linked to both normal morphology and good chromatin integrity of the spermatozoa in the original ejaculates [19], SLC-selected sperm doses should, theoretically, produce good pregnancy rates when used for AI. However, since factors other than sperm quality are involved in the fertility, it is often difficult to show an improvement in pregnancy rates under "standard" conditions, i.e. sperm doses from a normal stallion, processed and stored under optimal conditions, used for AI in normal mares by experienced personnel. A large sample size is needed to compensate for extraneous variables [20]. Such a fertility trial requires considerable time and resources and is beyond the scope of the present review. However, some initial observations have been made on the fertility of SLC-selected stallion spermatozoa, with particular reference to areas that are currently considered "challenging" in commercial horse breeding.

From the experimental observations listed above, several applications of SLC can be suggested, which are discussed below.

\section{Improvement of sperm quality from subfertile stallions}

Sperm doses from some "problem" stallions showed an improvement in fertility when SLC-selection was used, for example, pregnancy rates of $<20 \%$ without SLC can rise to $>50 \%$ after SLC, depending on the nature of the problem $[21,22]$, although the number of mares inseminated was small. The problems in four of the five stallions reported in the latter study were large volume with low sperm concentration with or without poor motility (two stallions), poor morphology (1 stallion), and one case of presumed ampullary stasis. Although sperm quality in the ejaculates from the first three stallions was demonstrably better, sperm morphology was still sub-optimal in the SLC-samples from the stallion with ampullary stasis since the

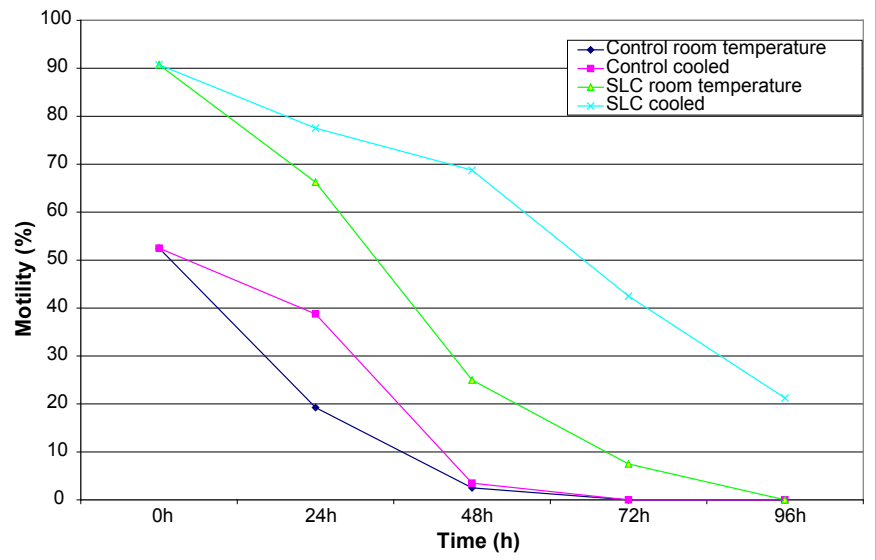

Note: $0 \mathrm{~h}$ is the time of semen collection

Figure 1: Effect on sperm motility of Single Layer Centrifugation of spermatozoa that do not normally tolerate cooling.

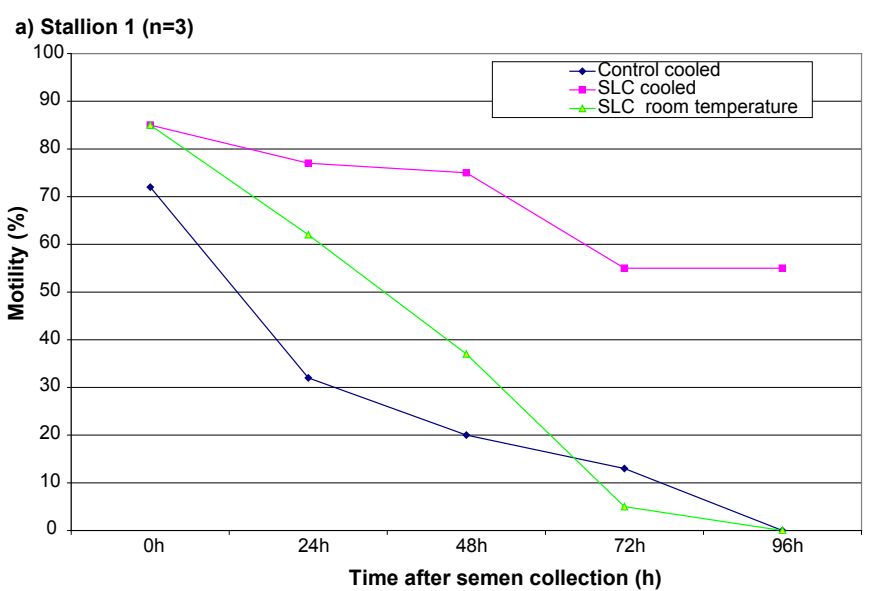

b) Stallion $2(n=3)$

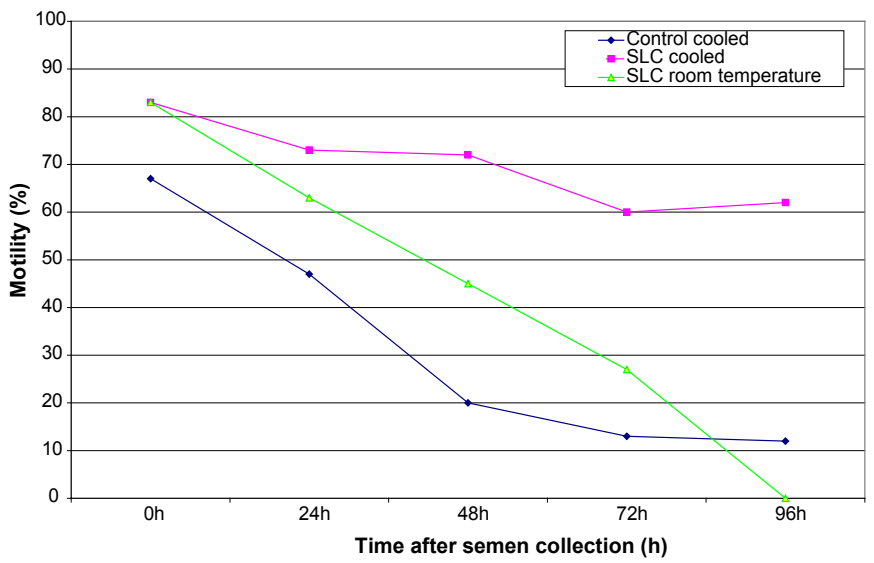

Figure 2: Retention of progressive motility in SLC-selected stallion sperm samples compared to uncentrifuged controls. Time 0 is the day of semen collection.

detached heads pelleted along with the normal spermatozoa. The chromatin damage was also increased in the SLC-samples compared to the uncentrifuged samples, which was in contrast to previous findings in a large number of stallions [14]. The presence of the detached heads may have been associated with the increased \%DFI in this sample.

\section{Spermatozoa that show low tolerance to cooling}

In some stallion ejaculates, progressive motility rapidly declines to less than $20 \%$ when the semen is extended and cooled. SLCselected sperm samples from such ejaculates showed a considerable improvement i progressive motility compared to the control samples (Figure 1). Thus two options are possible: SLC followed either by cooling to $4-6^{\circ} \mathrm{C}$ or storage at ambient temperature $\left(20^{\circ} \mathrm{C}\right)$.

Samples from four such stallions have been processed by SLC and used for AI: one was used for AI immediately after processing [22]; the other three were cooled and transported to another stud for AI after 24h. All four mares conceived, indicating that stallion spermatozoa with a low tolerance to cooling in the presence of some seminal plasma benefit from its complete removal.

When the pregnancy data from these four stallions were combined with those for the four "problem" stallions mentioned in the preceding section, an overall pregnancy rate of $57 \%$ (12 out of 21 inseminated 
mares) was obtained, which is close to the overall rate of $54 \%$ reported for AI with cooled transported semen in Sweden in the 2004 breeding season [23]

\section{Extending the shelf life of normal semen doses}

The common practice of collecting semen from stallions three times a week (Monday, Wednesday and Friday) causes some problems for both stallion and mare owners. Stallion owners may not be able to supply semen on every "standard" collection day, particularly from Sport horse stallion that may be competing. Furthermore, on any collection day, demand may exceed supply, particularly for popular stallions. Mare owners also face the difficulty of ordering semen in advance of ovulation, the perishable nature of the product and the availability of delivery services at weekends and public holidays. Therefore, a practical and efficacious method of prolonging the useable shelf-life of semen doses would have considerable application.

SLC offers several options to deal with these problems. First, progressive motility is prolonged in SLC-selected sperm samples, at least up to $72 \mathrm{~h}$ and often longer, depending upon the stallion. Motility sequences were obtained for multiple ejaculates from more than 50 stallions during the course of the project $[8,14]$, three of which are shown in (Figure 2). Progressive motility was assessed subjectively on a daily basis in samples stored in Kenney's extender at $6^{\circ} \mathrm{C}$ and at ambient temperature $\left(20^{\circ} \mathrm{C}\right.$ in this case $)$. In examples a and $\mathrm{b}$, progressive motility was still acceptable after $96 \mathrm{~h}$ cooled storage, although remained above $35 \%$ for only $48 \mathrm{~h}$ at room temperature, while it was still acceptable in the third stallion after $72 \mathrm{~h}$ storage at ambient temperature. In a different experiment, progressive motility was retained for longer and chromatin damage was reduced when INRA96 was used as a semen extender instead of Kenney's extender [15].

To test the hypothesis that the fertility of SLC-selected sperm doses is retained along with the progressive motility, SLC-selected sperm samples were inseminated into eight oestrous mares after $48-72 \mathrm{~h}$ storage at $6^{\circ} \mathrm{C}$. Conceptuses were identified by ultrasound examinatioin at 16-18 days after ovulation in 3 out of 4 mares inseminated with $48 \mathrm{~h}$ selected spermatozoa and 2 out of 3 of the mares inseminated with $72 \mathrm{~h}$ selected spermatozoa, indicating that SLC-selected spermatozoa are capable of achieving fertilization even after storage for up to $72 \mathrm{~h}$ at $6^{\circ} \mathrm{C}$ [24].

The second option suggested by the motility results shown in (Figure 2), is to store the SLC-selected spermatozoa at ambient temperature. This possibility is currently under investigation, particularly for stallions whose spermatozoa do not tolerate cooling.

The third option provided by SLC for extending the shelf-life of semen doses is to perform the SLC on cooled semen after transport to the stud where the mares are kept. Typically such insemination doses arrive after overnight transport, approximately $15-24 \mathrm{~h}$ after semen collection. Initial laboratory experiments using Androcoll-ESmall with semen samples extended in Kenney's extender showed variable results between stallions, with some stored ejaculates giving comparable SLC-preparations to fresh semen whereas others yielded very few spermatozoa in the pellet. However, using Androcoll-E-Large on semen from 15 stallions (three ejaculates per stallion), stored in INRA96, gave a good sperm yield from all stallions (median 50\%), with sperm quality comparable to SLC on fresh semen [25]. Furthermore, total motility, progressive motility and chromatin integrity (Table 1) were significantly improved in the SLC samples compared to the corresponding non-centrifuged samples $(\mathrm{P}<0.001)$, both immediately after SLC and after a further $24 \mathrm{~h}$ storage [25]. Normal morphology was also improved in the SLC samples compared to the uncentrifuged controls $(\mathrm{P}<0.01)$

\section{SLC and sperm cryopreservation}

Selecting the best spermatozoa prior to cryopreservation: SLCselected spermatozoa show enhanced post-thaw motility, viability, morphology and chromatin integrity [26]. The SLC-sperm preparations from stallions classified as poor freezers were of better quality than controls prepared by cushion-centrifugation. However, for stallions classified as "good freezers", the yield of sperm doses was reduced in the SLC-group. This reduction would be a considerable disadvantage unless a concomitant reduction in sperm dose could be achieved because of the improved sperm quality.

Selecting viable spermatozoa after cryopreservation: SLC can be used to select spermatozoa with good motility, viability and chromatin integrity post-thawing $[12,16]$. Furthermore, SLC-Large can be used to improve sperm quality in sub-standard batches of frozen stallion semen. In a preliminary study with eight batches of frozen semen that had been rejected on the basis of a subjective assessment of $>35 \%$ progressive motility, the motility (measured by the Qualisperm ${ }^{\text {TM }}$ Motility Analyzer) was increased by a mean of $13 \pm 10 \%$ and viability (measured with the Nucleocounter SP-100) by a mean of $9 \pm 7 \%$ (Stuhtmann \& Morrell, unpublished data). Seven of these eight samples would have been acceptable for AI after SLC. The fertility of these "rescued" batches will be tested in an AI trial.

Frozen-thawed SLC-selected spermatozoa functioned normally when injected into in vitro matured equine oocytes using intracytoplasmic sperm injection [27]. SLC showed some advantages over DGC when preparing sperm samples from two sub-fertile stallions for ICSI [28] in that the number of blastocysts was increased by almost $50 \%$ in the oocytes injected with SLC-selected spermatozoa

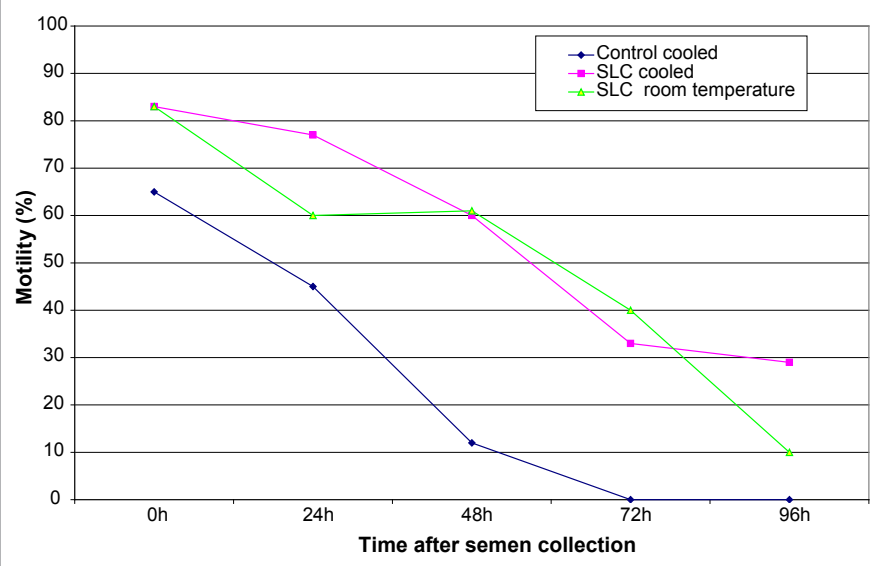

Figure 3: Stallion $3(n=3)$.

\begin{tabular}{|l|l|l|l|l|l|l|}
\hline Sample & \multicolumn{5}{|l|}{ Total Motility (\%) } & \multicolumn{2}{l|}{$\begin{array}{l}\text { Progressive motility } \\
(\%)\end{array}$} & $\begin{array}{l}\text { Normal } \\
\text { morphology (\%) }\end{array}$ & \%DF \\
\hline & $24 \mathrm{~h}$ & $48 \mathrm{~h}$ & $24 \mathrm{~h}$ & $48 \mathrm{~h}$ & & \\
\hline Control & 76.4 & 59 & 52.1 & 33 & 65.5 & 15.4 \\
\hline SLC & 82.6 & 68 & 59.6 & 46 & 70 & 13.0 \\
\hline & $\mathrm{P}<0.001$ & $\mathrm{P}<0.001$ & $\mathrm{P}<0.001$ & $\mathrm{P}<0.001$ & $\mathrm{P}<0.01$ & $\mathrm{P}<0.001$ \\
\hline
\end{tabular}

Note: \%DFI is the DNA fragmentation index from the Sperm Chromatin Structure Assay

Table 1: Effect of SLC on sperm quality in stored semen doses $(n=45)$. 
versus DGC-selected spermatozoa (21 versus 15 , respectively). A larger sample size would be required, however, to establish a statistical significance. In the absence of a reliable and repeatable method of performing conventional equine in vitro fertilization (IVF), ICSI is the only way to obtain offspring from some sub-fertile stallions and a way of making this labour-intensive procedure more efficient is potentially useful.

\section{Removal of pathogens}

Although all breeding stallions are tested for the presence of antibodies to EVA before they can be used for breeding, it is possible for stallions to shed the virus in semen and yet be sero-negative when tested for antibodies to the virus. Thus naive mares are at risk of infection via semen doses from shedding stallions. It has been shown previously that spermatozoa could be separated from equine arteritis virus in stallion semen using DGC followed by swim-up [29]. However, the volumes of semen that can be processed by such a method are limited (approximately $1.5 \mathrm{~mL}$ ), which would be impractical for processing equine semen doses. Recently, virus-free sperm samples were obtained from a shedding stallion by SLC with Androcoll-E, i.e. the SLC step alone appeared to sufficient to separate spermatozoa from virus in this case (JM Morrell, C Baule, M Wallgren \& G Blomquist, unpublished data). However, complete removal may depend on the initial level of virus in the ejaculate; further work is in progress to identify levels of virus that can be removed by SLC.

\section{Sexing spermatozoa}

A major problem with flow cytometric separation of $\mathrm{X}$ - and Y-chromosome bearing spermatozoa, first achieved by Morrell et al. [30] and subsequently by Johnson et al. [31], is the slow speed of the process and the limited yield of sexed spermatozoa that can be processed within a reasonable time. Thus the likelihood of achieving adequate numbers of sexed spermatozoa for conventional equine AI with flow cytometry alone is low. Furthermore, the flow cytometric technique is not applicable to all males and there appears to be a difference in "sortability" between individuals [32,34]. The latter authors attributed this variation in "sortability" to the number of dead spermatozoa present in the sample. Thus SLC could be used in conjunction with sperm sexing, to remove the dead and dying spermatozoa first, thus speeding up the process sufficiently to make it commercially viable $[32,33]$.

\section{Conservation of rare breeds}

Working with rare breeds, where the numbers of individuals available for breeding is reduced, poses several challenges. The small number of individuals may be closely related, in which case the changes of success are low, or they may be elderly or have low gamete quality. Since SLC has been shown to improve sperm quality in some problem stallions, it should be included as one of the potential tools to be used in trying to obtain offspring from these individuals.

\section{Conclusion}

SLC-selected spermatozoa have been shown to be capable of fertilization when used for AI in mares. The method has been used to improve the quality of some "problem" ejaculates, to extend the "shelflife" of sperm doses for AI, and to improve sperm survival during cryopreservation. Since spermatozoa can be separated from viruses and bacteria in semen by SLC, it may be possible to enhance the biosecurity of semen using this technique. SLC could also be potentially useful in conservation breeding and in sperm sexing by flow cytometry.

\section{Acknowledgements}

Sincere thanks are due to my colleagues who participated in these studies, particularly Heriberto Rodriguez-Martinez, Anders Johannisson, Anne-Marie Dalin, Johanna Lindahl, Gesa Stuhtmann and Susanne Meurling, and also to Prof. Twink Allen for reading the manuscript. The studies were funded by The Swedish Foundation for Equine Research, Stockholm, and the Lagerlöf Foundation, Stockholm, Sweden.

\section{References}

1. Morrell JM, Rodriguez-Martinez H (2009) Biomimetic techniques for improving sperm quality in animal breeding: a review. The Open Andrology Journal 1: 1-9.

2. Morrell JM, Rodriguez.Martinez H (2010) Practical applications of sperm selection techniques as a tool for improving reproductive efficiency. Vet Med Int.

3. Sharma RK, Seifarth K, Agarwal A (1997) Comparison of single- and two-layer Percoll Separation for selection of motile spermatozoa. Int J Fertil Womens Med 42: 412-417.

4. Sieme H, Martinsson G, Rauterberg H, Walter K, Aurich C, et al. (2003) Application of Techniques for Sperm Selection in Fresh and Frozen-Thawed Stallion Semen. Reprod Domest Anim 38: 134-140.

5. Morrell JM, Dalin A-M, Rodriguez-Martinez H (2008) Prolongation of stallion sperm survival by centrifugation through coated silica colloids: a preliminary study. Anim Reprod 5: 121-126.

6. Morrell JM, Johannisson A, Dalin AM, Rodriguez-Martinez H (2009a) Single Layer Centrifugation with Androcoll ${ }^{T w}-E$ can be scaled-up to allow large volumes of stallion ejaculate to be processed easily. Theriogenology 72: 879-884

7. Edmond AJ, Teague AR, Brinsko SP, Comerford KL, Waite JA, et al. (2008) Effect of density gradient centrifugation on quality and recovery of equine spermatozoa. Animal Reproduction Science 107: 318.

8. Morrell JM, Dalin A-M, Rodriguez-Martinez H (2009b) Comparison of density gradient and single layer centrifugation of stallion spermatozoa: yield, motility and survival. Equine Vet J 41: 53-58.

9. Morrell JM, Johannisson A, Dalin A-M, Rodriguez-Martinez H (2009c) Morphology and chromatin integrity of stallion spermatozoa prepared by density gradient and single layer centrifugation through silica colloids. Reprod Domest Anim 44: 512-517.

10. Morrell JM, Johannisson A, Strutz H, Dalin A-M, et al. (2009d) Colloida centrifugation of stallion semen: changes in sperm motility, velocity and chromatin integrity during storage. Journal Equine Veterinary Science 29: 24 32.

11. Johannisson A, Morrell JM, Thorén J, Jonsson M, Dalin AM, et al. (2009) Colloidal centrifugation with Androcoll- $\mathrm{E}^{\mathrm{TM}}$ prolongs stallion sperm motility, viability and chromatin integrity. Anim Reprod Sci 116: 119-128.

12. Macias Garcia B, Morrell JM, Ortega Ferrusola C, Fernandez-Gonzalez L, Tapia $\mathrm{JA}$, et al. (2009) Centrifugation on a single layer of colloid selects improved quality spermatozoa from frozen-thawed stallion semen. Reproduction Domestic Animals 44: 523-526.

13. Bergqvist AS, Johannisson A, Bäckgren L, Dalin AM, Rodriguez-Martinez $\mathrm{H}$, et al. (2011) Single layer centrifugation of stallion spermatozoa through Androcoll ${ }^{\mathrm{TM}}-\mathrm{E}$ does not adversely affect their capacitation-like status, as measured by CTC-staining. Reprod Domest Anim 46: 74-78.

14. Morrell JM, Rodriguez-Martinez H, Johannisson A (2010a) Single Laye Centrifugation of stallion spermatozoa consistently selects the most robust spermatozoa from the rest of the ejaculate in a large sample size: data from 3 breeding seasons. Equine Veterinary Journal 42: 579-585.

15. Morrell JM, Johannisson A, Juntilla L, Rytty K, Bäckgren L, et al. (2010b) Stallion sperm viability, as measured by the Nucleocounter SP-100, is affected by extender and enhanced by Single Layer Centrifugation. Vet Med Int.

16. Morrell JM, Rodriguez-Martinez H, Johannisson A (2010c) Single Layer Centrifugation of stallion spermatozoa improves sperm quality compared to sperm washing. Reprod Biomed Online 21: 429- 436.

17. Macias GB, Fernandez-Gonzalez L, Morrell J, Ortega Ferrusola C, Tapia JA et al. (2009) Single layer centrifugation through colloid positively modifies the sperm sub-population structure of frozen-thawed stallion spermatozoa. Animal Reproduction Science 114: 193-202. 
Citation: Morrell JM (2011) Biomimetics in Action: Practical Applications of Single Layer Centrifugation for equine breeding. J Veterinar Sci Technol 2:107. doi:10.4172/2157-7579.1000107

Page 5 of 5

18. Morrell JM, Wallgren M (2011) Removal of bacteria from boar ejaculates by Single Layer Centrifugation can reduce the use of antibiotics in semen extenders. Anim Reprod Sci 123: 64-69.

19. Morrell JM, Johannisson A, Dalin A-M, Hammar L, Sandebert T, et al. (2008) Sperm morphology and chromatin integrity on Swedish warmblood stallions and their relationship to pregnancy rates. Acta Vet Scand 50: 2.

20. Amann RP (2005) Weaknesses in reports of "fertility" for horses and other species. Theriogenology 63: 698-715.

21. Mari G, lacono E, Kutvölgyi G, Mislei B, Rodriguez-Martinez H, et al. (2010) Stallion spermatozoa prepared by Single Layer Centrifugation with Androcoll-E are capable of fertilisation in vivo. Reprod Dom Anim 45: 97.

22. Morrell JM, Mari G, Kutvölgyi G, Meurling S, lacono E, et al. (2010d) Spermatozoa from stallion ejaculates processed by Single Layer Centrifugation with Androcoll ${ }^{\mathrm{TM}}$-E. Reprod Domest Anim 46: 642-645.

23. Dahlsten A (2006) (Fertilitet hos svenska halvblodshingstar betäckningssäsongen 2004 - en pilotstudie. Examensarbete vid fakulteten för veterinärmedicin och husdjursvetenskap, SLU, veterinärprogrammet, sidor 1-25). Fertility in Swedish halfblood stallions in the 2004 breeding season preliminary study. Final year project at the Faculty of Veterinary Medicine and Animal Science, SLU, pp1-25

24. Lindahl J, Dalin A-M, Morrell JM (2011) Pregnancies in mares inseminated with spermatozoa selected by Single Layer Centrifugation and stored for $48 \mathrm{~h}$ or 72h. Reproduction Domestic Animals In press

25. Morrell JM, Macias Garcia B, Pena FJ, Johannisson A (2011) Processing stored stallion semen doses by Single Layer Centrifugation. Theriogenology [Epub ahead of print].
26. Hoogewijs M, Morrell JM, Van Soom A, Govaere J, Johannisson A, et al. (2011) Sperm selection using single layer centrifugation prior to cryopreservation can increase post thaw sperm quality in stallions. Equine Vet Journal, In Press.

27. Morrell JM, Colleoni S, Lagutina I, Rodriguez-Martinez H, Lazzari G, et al (2010e) Stallion spermatozoa selected by Single Layer Centrifugation with Androcoll ${ }^{\mathrm{TM}}$-E have normal functionality after ICSI. Animal Reproduction Science 121S: S196-S197

28. Colleoni S, Lagutina I, Rodriguez-Martinez H, Lazzari G, Galli C, et al. (2011) New techniques for selecting spermatozoa for equine assisted reproduction. JEVS, In press.

29. Morrell JM, Geraghty RM (2006) Effective removal of equine arteritis virus from stallion semen. Equine Vet J 38: 224-229.

30. Morrell JM, Keeler KD, Noakes DE, Mackenzie NM, Dresser DW (1988) Sexing of sperm by flow cytometry. Veterinary Record 122: 322-324.

31. Johnson LA, Flook JP, Hawk HW (1989) Sex preselection in rabbits: live births from $X$ and $Y$ sperm separated by DNA and cell sorting. Biol Reprod 41: 199203.

32. Underwood SL, Bathgate R, Ebsworth M, Maxwell WM, Evans G (2010) Pregnancy loss in heifers after artificial insemination with frozen-thawed, sexsorted, re-frozen-thawed dairy bull sperm. Anim Reprod Sci 118: 7-12.

33. Hollinshead FK, O’Brien JK, Maxwell WMC, Evans G (2004) Assessment of in vitro sperm characteristics after flow cytometric sorting of frozen-thawed bull spermatozoa. Theriogenology 62: 958-968.

34. Clulow JR, Evans G, Morris LHA, Maxwell WM (2009) Factors affecting the "sortability" of stallion spermatozoa into $\mathrm{X}$ - and Y-chromosome bearing populations. Animal Reproduction Science 113: 220-228. 